\title{
Feeding of deoxynivalenol increases the intestinal paracellular permeability of broiler chickens
}

\author{
Wageha A. Awad ${ }^{1} \cdot$ Daniel Ruhnau $^{1} \cdot$ Claudia Hess $^{1} \cdot$ Barbara Doupovec $^{2} \cdot$ Dian Schatzmayr $^{2} \cdot$ Michael Hess $^{1}$
}

Received: 20 February 2019 / Accepted: 18 April 2019 / Published online: 27 April 2019

(c) The Author(s) 2019

\begin{abstract}
In recent years, the deleterious effects attributed to mycotoxins, in particular on the intestine, faced increased attention and it was shown that deoxynivalenol (DON) causes adverse effects on gut health. In this context, it has been repeatedly reported that DON can alter the intestinal morphology, disrupt the intestinal barrier and reduce nutrient absorption. The underlying mechanism of a compromised intestinal barrier caused by DON in chickens has yet to be illustrated. Although, DON is rapidly absorbed from the upper parts of the small intestine, the effects on the large intestine cannot be excluded. Additionally, a damaging effect of DON on the gut epithelium might decrease the resistance of the gut against infectious agents. Consequently, the objectives of the present studies were: (1) to investigate the impact of DON on the epithelial paracellular permeability by demonstrating the mucosal to serosal flux of ${ }^{14} \mathrm{C}$-mannitol in the small and large intestine applying Ussing chambers and (2) to delineate the effects of DON on the colonization and translocation of Escherichia coli. Both parameters are well suited as potential indicators for gut barrier failure. For this, a total of 75 one-day-old Ross 308 broiler chickens were housed in floor pens on wood shavings with feed and water provided ad libitum. Birds were randomly allocated to three different groups ( $n=25$ with 5 replicates/group) and were fed for 5 weeks with either contaminated diets ( 5 or $10 \mathrm{mg}$ DON/kg feed) or basal diets (control). Body weight (BW) and BW gain of birds in the group fed with $10 \mathrm{mg} / \mathrm{kg}$ DON were significantly lower than in group with $5 \mathrm{mg} / \mathrm{kg}$ DON and the control group. Moreover, the mannitol flux in jejunum and cecum was significantly $(P<0.05)$ higher in DON-fed groups compared to control birds. Consistent with this, DON enhanced the translocation of $E$. coli with a higher number of bacteria encountered in the spleen and liver. Altogether, the actual results verified that DON can alter the intestinal paracellular permeability in broiler chickens and facilitates the translocation of enteric microorganisms such as E. coli to extra-intestinal organs. Considering that moderate levels of DON are present in feed, the consumption of DON-contaminated feed can induce an intestinal breakdown with negative consequences on broiler health.
\end{abstract}

Keywords DON $\cdot$ Broiler chickens $\cdot$ Mannitol flux $\cdot$ Permeability $\cdot$ Escherichia coli translocation

\section{Introduction}

Deoxynivalenol (DON) is the most common trichothecene mycotoxin detected in feedstuffs globally. Nonetheless, the development of agricultural and manufacturing practices, DON contamination cannot be avoided and represents a

Wageha A. Awad

wageha.awad@vetmeduni.ac.at

1 Department for Farm Animals and Veterinary Public Health, Clinic for Poultry and Fish Medicine, University of Veterinary Medicine, Veterinärplatz 1, 1210 Vienna, Austria

2 BIOMIN Research Center, Technopark 1, 3430 Tulln, Austria permanent health risk for both animals and humans, since it was indicated that DON affects not only the intestine but also other tissues and systems, including immune system, endocrine system and nervous system in humans and animals (Maresca 2013). The effects of DON exposure on different animal species are related to the concentration and duration of exposure as described in several reviews (Awad et al. 2010, 2012a, 2013; Grenier and Applegate 2013; Pinton and Oswald 2014). Such authors concluded that exposure to DON can compromise gut health for two main reasons. Firstly, the gut epithelium is repeatedly exposed to DON at higher concentrations than other tissues (Awad et al. 2007a, 2011; Ghareeb et al. 2013, 2015). Secondly, the gut epithelium consists of rapidly dividing cells with a high protein turnover (Osselaere et al. 2013) and these cells are the main targets of DON's 
inhibitory action on protein synthesis. Due to the damaging effect of DON on the gut epithelium, it was hypothesized that the toxin decreases the resistance of the gut to infectious agents (Vandenbroucke et al. 2011; Antonissen et al. 2014).

Overall, the intestinal epithelium acts as a selectively permeable barrier (physical), permitting the passage of nutrients, water, and ions and at the same time restrains noxious substances and microorganisms within the gut (Awad et al. 2015, 2018). Thus, intestinal integrity is critical for maintaining a physical barrier between the intestinal lumen and the body to protect against dissemination of pathogens. It was reported that the intestinal permeability can be defined as a quantifiable indicator for the intestinal barrier at given sites, via analyzing flux rates or uptake of defined molecules (e.g., electrolytes or sugars) across the intestinal epithelium by the Ussing chamber technique. In general, it is important to understand the pathophysiological pathways and mechanisms that influence the intestinal barrier, since a disrupted barrier function is associated with an increased epithelial permeability and translocation of luminal antigens (e.g., pathogens, toxins), as well as a non-specific inflammatory response (Groschwitz and Hogan 2009; Odenwald and Turner 2013; Awad et al. 2017).

The toxic effects of DON on the intestine are mediated by various mechanisms such as down-regulation of nutrient transporters, decrease in nutrient absorption and reduce of tissue resistance. DON also affects intestinal health by altering goblet cells functions such as: (1) the production of intestinal mucus that plays an important role in the gut barrier function and (2) the production of trefoil factors that contribute to the renewal/healing of the epithelium (Pinton et al. 2015; Graziani et al. 2019). Although many studies have evaluated the effects of DON on the small intestine of broilers, there is a lack of information on the effects of DON on the large intestine. In addition, the contribution of DON to the loss of intestinal barrier function has not been extensively investigated in chickens. A disruption of the intestinal barrier was reported in mice challenged orally with DON ( $25 \mathrm{mg} / \mathrm{kg}$ bw) demonstrating a significant increase in paracellular transport of FITC-dextran (Akbari et al. 2014). However, in chickens, such an effect of DON on the paracellular pathway and its consequences on bacterial translocation has not been investigated so far. Therefore, the aim of the study was (1) to verify an effect of DON on the intestinal barrier by measuring ${ }^{14} \mathrm{C}$-Mannitol fluxes in chickens applying the Ussing chamber technique and (2) to determine the translocation of $E$. coli to internal organs.

\section{Materials and methods}

\section{Ethics statement}

Animal trial was approved by the institutional ethics committee of the University of Veterinary Medicine and the
Ministry of Research and Science under the license number GZ 68.205/0159-WF/V/3b/2017.

\section{Birds and feeding}

A total of 75 one-day-old broiler chickens (males and females) were obtained from a commercial hatchery (Ross308, Geflügelhof Schulz, Graz, Austria) and randomly divided into three groups ( 25 birds $/ 5$ replicates/group). The birds were housed on wood shavings and were provided with their diets and water ad libitum. The broilers were fed for 5 consecutive weeks with either contaminated diets ( 5 or $10 \mathrm{mg}$ DON$/ \mathrm{kg}$ feed) or basal diets (control) based on maize, wheat, soybean meal, soybean oil, soya and rapeseed oil, and a premix with vitamins, minerals, amino acids, salt, and mono-calcium phosphate. Chicks were fed the starter diet from day 1 to day 10 , and a grower diet from day 11 to day 35. Representative feed samples for each group were analyzed for determining the concentration of DON and other mycotoxins in the diets. Until the end of animal trials, the birds were monitored daily for any clinical signs.

\section{Body weight of birds}

Body weight (BW) was determined in weekly intervals and the body weight gain (BWG) was calculated as the difference between the final and initial bird weight during each of the weighing periods. Furthermore, feed intake over the course of the experiment was measured and consequently feed conversion ratio (FCR) was calculated per pen. At each time point (21, 28, and 35 days of age), 5 birds from each group were killed by bleeding of the jugular vein under anaesthesia with a single dose of thiopental $(1 \mathrm{mg} / \mathrm{kg})$ injection into the wing vein and samples from jejunum, cecum, liver and spleen tissues were collected for E. coli enumeration using the method described below. Furthermore, at the end of the feeding trial, ten birds from each group were killed for Ussing chamber measurements.

\section{Determining the paracellular permeability by Ussing chamber technique}

At the end of the feeding trial, isolated epithelia were incubated in Ussing chambers as described by Awad et al. (2014a). The Ussing chamber is connected to a mucosal and serosal buffer reservoir that allows the separate incubation of the mucosal and serosal sides in aerated buffer solution. Flux rates of mannitol $\left(J_{\operatorname{man}}\right)$, were measured at a bilateral concentration of $10 \mathrm{mM}$. The radioactive tracer, ${ }^{14} \mathrm{C}$-mannitol $(0.1 \mathrm{mCi} / \mathrm{ml}$; Hartmann Analytic), was added to the mucosal solution. After a 30-min equilibration period, standards were taken from the mucosal side of each chamber and a 30-min flux period was established by taking $0.6-\mathrm{mL}$ samples from 
the serosal compartment (Aschenbach et al. 2000; Tavelin 2003). Epithelia were incubated for about $3 \mathrm{~h}$ divided into three flux periods which allows to evaluate the effect of DON (baseline measurement period) and subsequent exposure to mucosal hyperosmolarity (challenge period, increasing the luminal mannitol concentration from 10 to $20 \mathrm{mM}$ ) and persistent effects of the hyperosmolarity challenge (persistent period). The presence of ${ }^{14} \mathrm{C}$ mannitol was established by measuring $\beta$ emission in a liquid scintillation counter after addition of a liquid scintillation fluid (Aquasafe 300 Plus, Zinsser Analytic, Maidenhead, UK). Unidirectional ${ }^{14} \mathrm{C}$ mannitol fluxes from mucosa to serosa $\left(J_{\mathrm{ms}}\right)$ were evaluated by calculating the net appearance of ${ }^{14} \mathrm{C}$ overtime in the serosal bathing solution as a ratio of flux/concentration as described previously (Aschenbach et al. 2000).

\section{Bacterial enumeration}

For quantification of $E$. coli in the intestine (jejunal and cecal content) or liver and spleen, $1 \mathrm{~g}$ of feces/organ material was collected from ten birds per group at each killing time point, and diluted 1:10 (wt:vol) in phosphate-buffered saline (PBS) (BR0014G, OXOID, Hampshire, UK). The mixture was homogenized using an Ultra-Turrax (IKA, Staufen, Germany). Afterwards, serial tenfold dilutions were made from each sample, and $100 \mu \mathrm{l}$ from each dilution were directplated in duplicate onto MacConkey agar (Scharlau, Barcelona, Spain). The plates were incubated at $37{ }^{\circ} \mathrm{C}$ for $24 \mathrm{~h}$. After incubation, E. coli colonies were counted as colonyforming units per gram.

\section{Statistical analysis}

Data are presented as means with SEM. Following tests for normality (Kolmogorov-Smirnov's test), statistical analysis of performance, bacterial translocation and mannitol fluxes data for significant differences between the three groups were performed using a multivariate general linear model and Duncan's multiple range test. Differences were considered significant at a level of $P \leq 0.05$. All tests were performed using IBM SPSS statistics 24, SPSS software (Chicago, IL, USA).

\section{Results}

\section{DON-associated change in body weight}

Growth performance of broiler chickens, in terms of the average body weight, was numerically decreased $(P<0.1)$ at day 7 of age birds fed with 10 ppm DON (DON 10) compared to the other groups. Later, at days 14, 21 and 28 of age, the body weight was significantly depressed by feeding of
DON 10 compared to the other groups $(P<0.05$, Fig. 1$)$. The weekly BWG was also decreased by DON treatments. However, this effect was especially pronounced at the beginning of the experiment and decreased thereafter. In addition, the level of feed intake was generally lower in broilers fed DONcontaminated diets (Fig. 2) and it significantly decreased by increasing the DON concentrations in diets. In contrast, at early stage, the feed conversion rate (FCR, Fig. 2) was higher in DON-10-fed birds compared with control birds which indicates that DON could affect feed efficiency under the present experimental conditions. Later on, the effects on performance were compensated, as the significant differences between groups decreased by age.

\section{DON-associated changes in intestinal permeability}

To elucidate whether the paracellular epithelial permeation pathway is altered by DON, the fluxes of a known paracellular marker molecule, mannitol, were used. The unidirectional mucosa-to-serosa permeability of mannitol in jejunum and cecum is shown in Fig. 3. The results
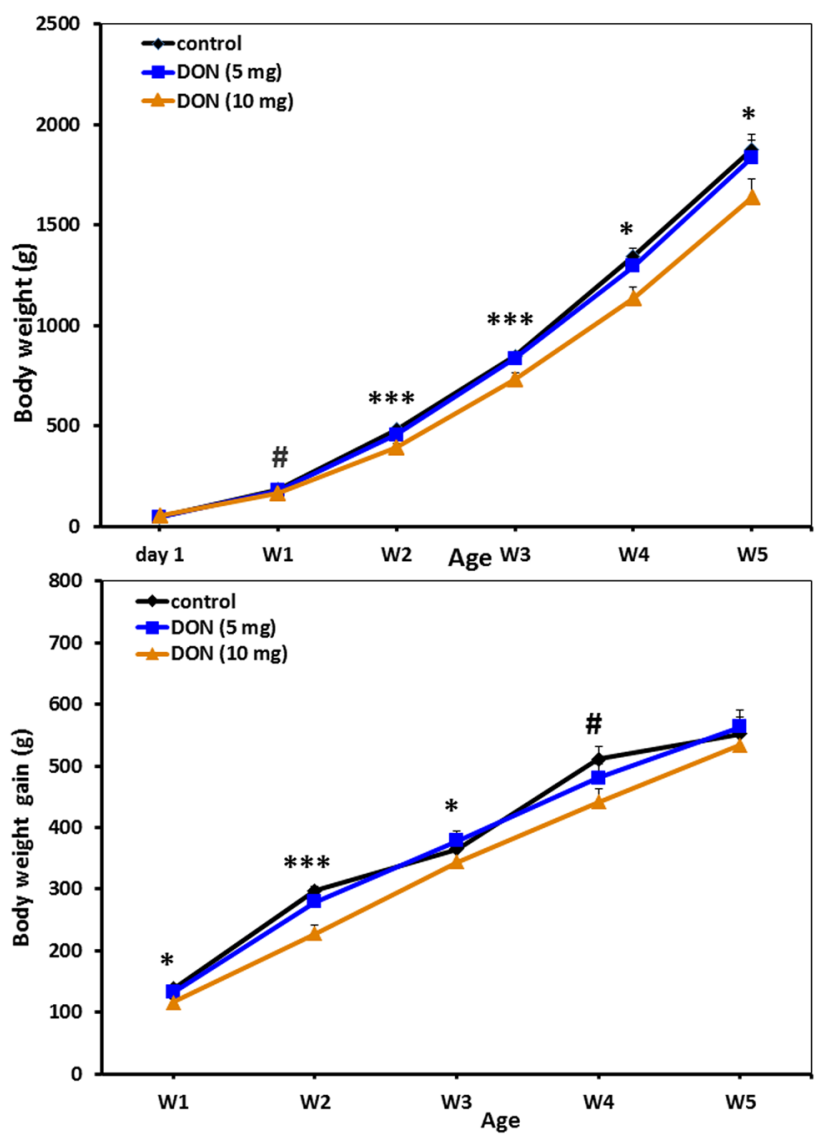

Fig. 1 Effect of DON-contaminated feed on body weight ( $\mathrm{g}$ ) and body weight gain (BWG) of broiler chickens. Results are presented as mean values and SEM $(n=25)$. Asterisks mark differences with $P \leq 0.1\left(^{\#}\right), P \leq 0.05\left(^{*}\right)$, or $P \leq 0.001$ (***) 

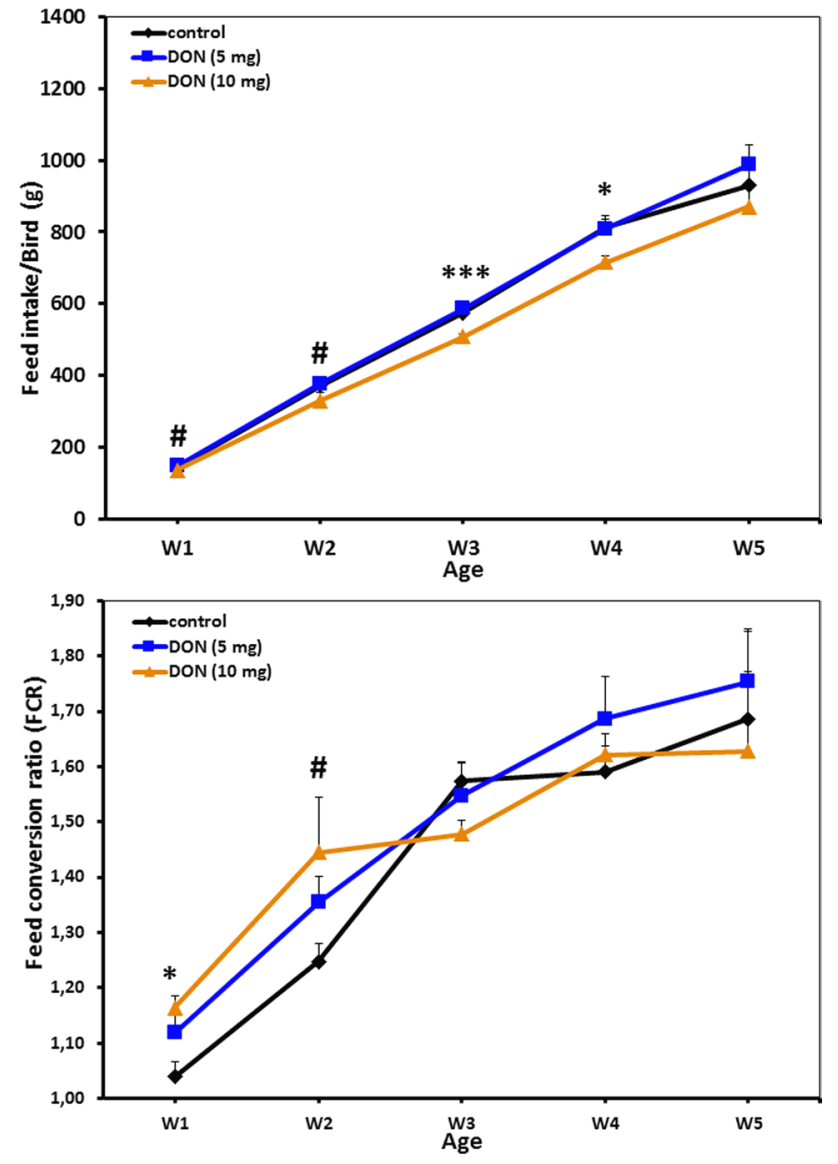

Fig. 2 Effect of DON-contaminated feed on feed intake (g) and feed conversion ratio of broiler chickens. Results are presented as mean values and SEM $(n=25)$. Asterisks mark differences with $P \leq 0.1\left(^{\#}\right)$, $P \leq 0.05(*)$, or $P \leq 0.001(* * *)$

revealed that DON exposure induces a significant increase in the flux of mannitol in the jejunum in a dose-dependent manner in all flux periods, indicating an increased paracellular leakage. During the baseline period, there were no differences in the flux of mannitol in the cecum among different groups. However, during the challenge period (the second flux period), the increased luminal mannitol concentration from 10 to $20 \mathrm{mM}$ resulted in an immediate increase in mannitol flux in a non-dose-dependent manner, most probably due to passive diffusion considering that paracellular is much more conductive than the transcellular route. Nevertheless, the effect of DON 10 was more persistent in the cecum compared with DON 5, as there was a significant difference at later time-points. In addition, statistical reliable differences $(P<0.001)$ in paracellular permeability were found among jejunum and cecum in the control and DON groups, as the fluxes of mannitol were higher in the cecum than jejunum.
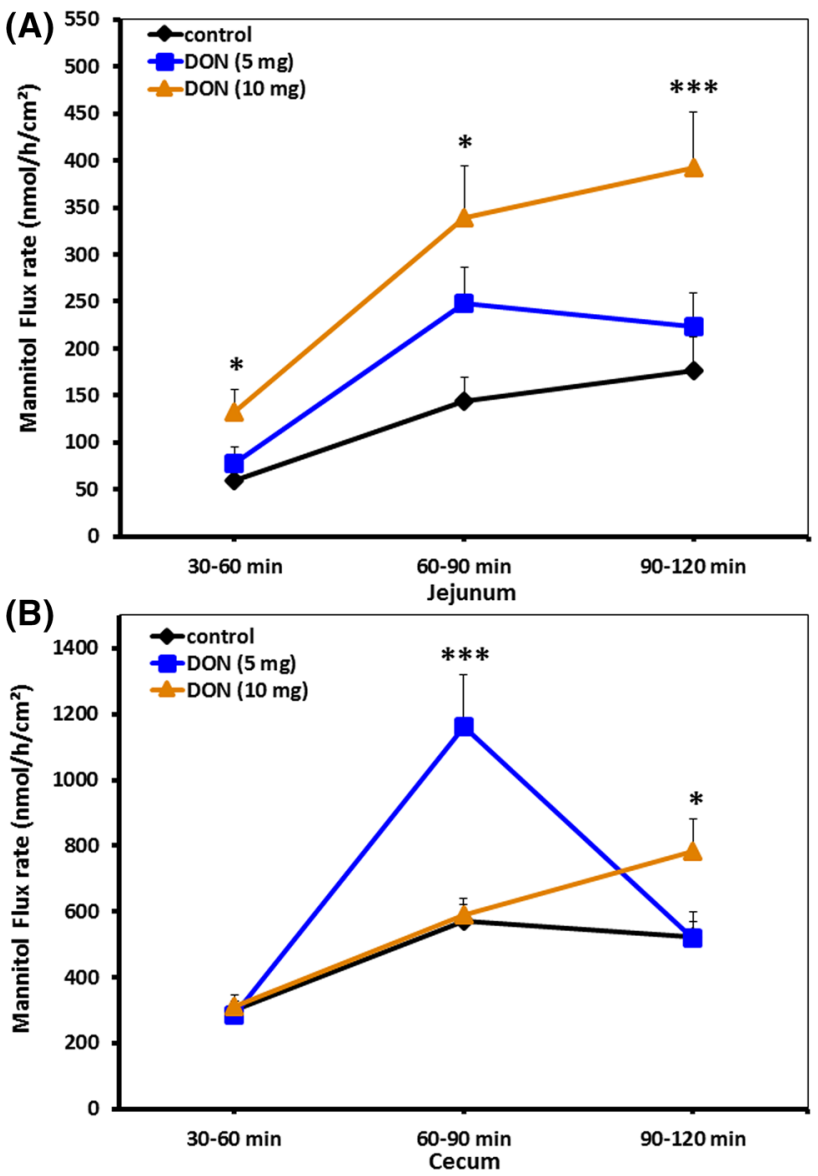

Fig. 3 Effect of DON-contaminated feed on paracellular permeability in jejunum (a) and cecum (b). Mucosal to serosal flux $\left(J_{\mathrm{ms}}\right)$ of the permeability marker ${ }^{14} \mathrm{C}$-mannitol were performed in Ussing chambers. Data are presented as the mean values and SEM $(n=10)$. Asterisks mark differences with $P \leq 0.1\left(^{*}\right), P \leq 0.05(*)$, or $P \leq 0.001(* * *)$

\section{Influence of DON on the colonization and translocation of $E$. coli}

The $E$. coli enumeration in the digesta content of the small and large intestine is presented in Fig. 4. In DON-fed groups, birds had higher $E$. coli loads in the jejunum and cecum at day 21 compared to the controls; however at that time, no significant differences were found regarding the $E$. coli counts in the liver and spleen of the birds fed different diets. In addition, at day $28, E$. coli counts were higher in the liver and spleen of DON-fed birds compared to the controls. Moreover, the E. coli translocation was not linearly increased with higher DON concentration, as no significant difference was observed between DON 5 and DON 10 except that the E. coli translocation to the spleen at day 28 was higher of DON-5-fed birds compared to the DON-10fed birds. These data demonstrated that $E$. coli translocation to the liver and spleen increased in DON-fed birds during the later stages of exposure. The results provided clear evidence 

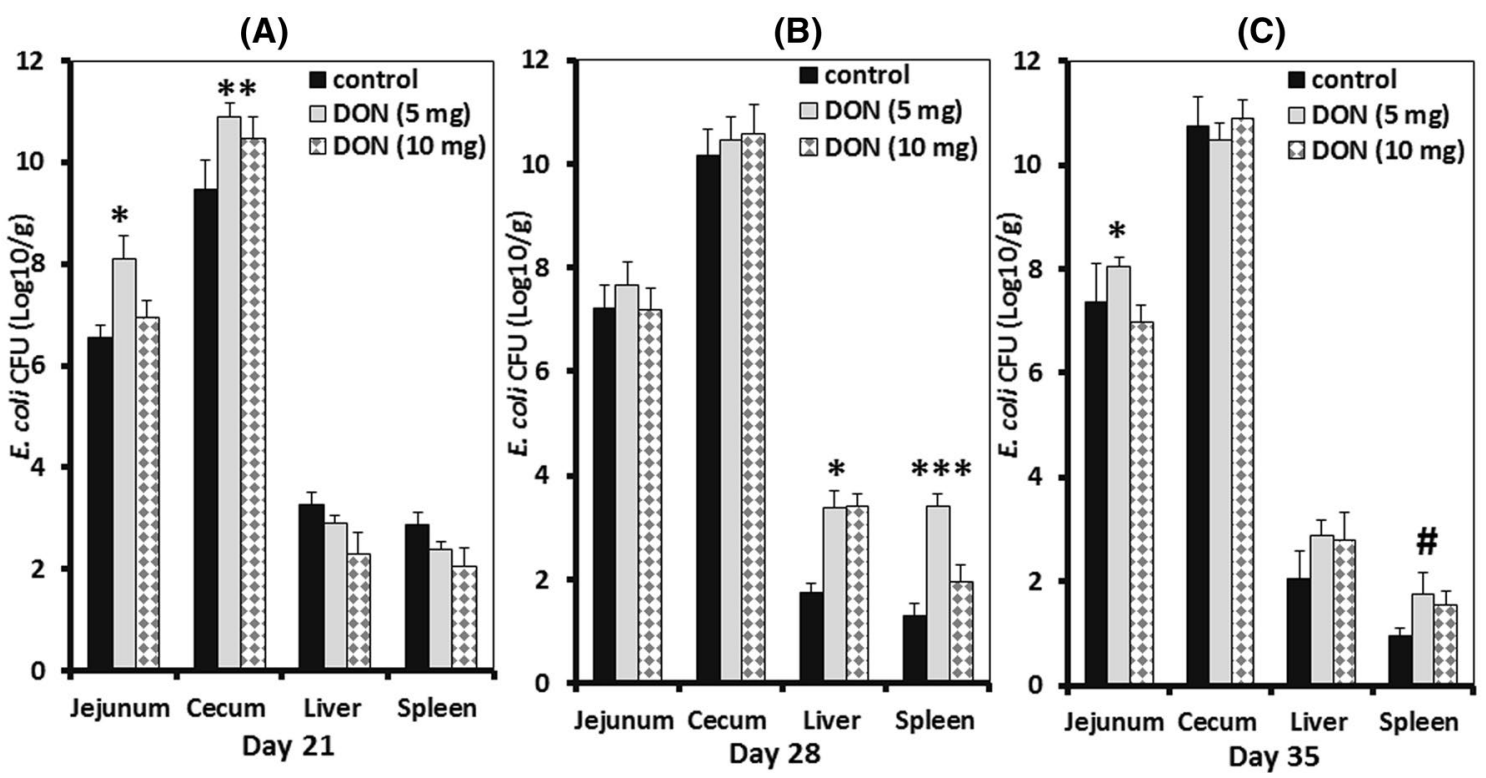

Fig. 4 E. coli counts in the jejunum and cecum of birds fed with and without DON-contaminated feed. Results are presented as mean values and SEM $(n=5)$. Numbers of bacteria are expressed in logarith-

mic form of colony-forming units (log CFU/g). Asterisks mark differences with $P \leq 0.1\left(^{\#}\right), P \leq 0.05\left(^{*}\right), P \leq 0.01\left(^{* *}\right)$, or $P \leq 0.001\left(^{* * *}\right)$

that DON increased the paracellular intestinal permeability and elevated the $E$. coli translocation to the liver and spleen. This suggests that the mechanism for bacterial translocation after DON feeding involves alterations of the paracellular permeability of the gut.

\section{Discussion}

The contamination of cereal-based products by mycotoxins is an emerging issue worldwide with a considerable impact on animal and human health. In poultry production, the contamination of feed with DON is of great concern due to the high exposure level resulting from cereal-rich diets. The molecular mode of action of DON depends on its ability to inhibit protein, RNA and DNA synthesis and to induce a 'ribotoxic stress response' characterized by the activation of mitogen-activated protein kinases (MAPKs) (Awad et al. 2014a, b; Ghareeb et al. 2016).

Due to the importance of the gastrointestinal tract as an initial organ to be encountered after the intake of DONcontaminated feed, alterations of the intestinal barrier due to exposure of DON have been addressed in several studies. It has been repeatedly reported that DON can induce negative impacts on gut health, such as alteration of the intestinal morphology (Awad et al. 2006a, b, Awad et al. 2011a, b; Kolf-Clauw et al. 2009), disruption of the intestinal barrier function via increasing tissue conductance (Awad et al. 2004, 2005a, b; Pinton et al. 2009; Awad and Zentek 2015), reduction of nutrient absorption (Maresca et al. 2002; Awad

et al. 2007a, 2011b), production of intestinal mucus that play an important role in the gut barrier function, production of trefoil factors that contribute to the renewal/healing of the epithelium (Pinton et al. 2015; Graziani et al. 2019) and modulation of the tight-junction proteins expression (Pinton et al. 2009; Osselaere et al. 2013). All those results indicate that DON can, indeed, alter intestinal barrier functions with direct or indirect negative consequences for production efficiency.

Previous studies indicated that the impact of DON on poultry performance was quite contradictory compared to that in other animals, due to differences in chicken breeds and dietary composition (Kubena et al. 1988, 1989; Dänicke et al. 2003; Awad et al. 2004, 2006a; Ghareeb et al. 2014). In the current study, however, DON levels of $10 \mathrm{mg} / \mathrm{kg}$ had a stronger inhibiting effect on the growth of chickens compared with $5 \mathrm{mg} / \mathrm{kg}$, as weight differences were more pronounced between 5 and $10 \mathrm{mg} / \mathrm{kg}$ DON groups. These results are in agreement with previous studies, demonstrating that feeding of DON-contaminated diets compromised the growth performance of chickens (Dänicke et al. 2003; Andretta et al. 2011; Awad et al. 2012b).

Besides, more insights into implications of the long-term effects of DON consumption on intestinal permeability in broilers were provided in the current study. The measurement of tissue resistance is considered as a good indicator of the integrity of the epithelial barrier. This decrease could indicate an alteration in the transcellular or paracellular ion flux. Accordingly, to identify that, we evaluated the mucosal to serosal flux of a paracellular marker (mannitol) 
in jejunum and cecum, as less is known about the intestinal site-dependent effects of DON in broilers. The results revealed, for the first time, that DON increased the fluxes of mannitol in jejunum in a dose-dependent manner. Furthermore, this effect became more prominent in the cecum even with a low dose of DON, which might be explained by indirect effects of DON due to changes in cecal nutrient flow and mucus secretion (Antonissen et al. 2015). In addition, the fluxes of mannitol were higher in the cecum than jejunum, as the cecum is the intestinal segment in which the paracellular permeability is quite significant which was also confirmed in the actual study.

In this regard, Amat et al. (1999) showed that medial and distal segments of the ceca are moderately tight regions, indicating that the relative leakiness of the chicken cecum differs from the intestine of mammals (Awad et al. 2007b). Furthermore, in chickens, the highest number of microbiota is found in the cecum and plays a crucial role in modulation of the gut epithelial barrier (Awad et al. 2016a, b). Moreover, the longer retention time of digesta in the ceca permits a complete microbial breakdown of complex fiber and in consequence enhances short-chain fatty acids (SCFAs) production (Oakley et al. 2014). Furthermore, alterations in SCFAs concentrations might increase translocation of enteric pathogenic bacteria to extra-intestinal sites by affecting the virulence of bacteria via providing a signal for expression of invasion genes (Lawhon et al. 2002).

Concurrently, the presented data clearly showed that DON can increase gut paracellular permeability. Furthermore, it was hypothesized that an increased intestinal permeability induced by DON in chickens might promote the translocation of other enteric microorganisms. Evidence from previous studies indicated that DON exposure in the intestines render the intestinal epithelium more susceptible to enteric infections, translocation and also potentiate the inflammation. In this context, the translocation of a pathogenic $E$. coli across the porcine IPEC-1 epithelial cells increased in a dose-dependent manner following DON exposure (Pinton et al. 2009). Of great concern, however, is the result that also low non-cytotoxic concentrations of DON seem to be able to affect this intestinal barrier function, leading to the translocation of bacteria such as E. coli in human intestinal Caco-2 cells (Maresca et al. 2008). Similarly, a higher translocation of Salmonella typhimurium was also observed in porcine IPEC-J2 exposed to low doses of DON (Vandenbroucke et al. 2011). For validation and extension of those findings, we investigated the specific relation between the increase of intestinal paracellular permeability and bacterial translocation.

Apart from confirming permeability changes in the intestine of broiler chickens, this study revealed, for the first time, that DON initially increased the E. coli loads in the gut and then promoted the translocation of $E$. coli with a higher number encountered in the liver and spleen. Such findings are in agreement with Pinton et al. (2009) and Maresca et al. (2008) who found that DON promotes the translocation of $E$. coli across the intestinal epithelium of porcine and humans. The results indicate that the effect of DON on bacterial translocation could be correlated with the ability of this toxin to specifically increase the paracellular permeability. This could also be promoted by alterations in SCFAs concentrations as such changes promote the translocation of enteric bacteria to extra-intestinal sites by increasing the expression of invasion genes (Lawhon et al. 2002).

Taken together, the results of the actual study indicate that ingestion of a DON-contaminated diet exacerbates the intestinal paracellular permeability. Furthermore, DON can alter gut colonization dynamics of certain microorganisms as demonstrated for E. coli. In addition, DON promotes the translocation of luminal bacteria with possible consequences on animal health. The presented results showed that the applicable European guidance value of $5 \mathrm{mg}$ DON $/ \mathrm{kg}$ feed can influence gut health in broiler chickens. Therefore, permissible levels of DON contamination need to be re-thought as potential stress factor for gut health in broilers.

Acknowledgements Open access funding provided by University of Veterinary Medicine Vienna. This work was financed by the Austrian Research Promotion Agency (FFG; project number 858543) and Biomin Holding GmbH (Technopark 1, 3430 Tulln, Austria). We would like to thank A. Georgi, D. Jandreski-Cvetkovic for their technical contributions, and V. Stanisavljevic and A. Sandor for their assistance during the animal trial.

Conflict of interest The authors declare that they have no conflict of interest.

Open Access This article is distributed under the terms of the Creative Commons Attribution 4.0 International License (http://creativeco mmons.org/licenses/by/4.0/), which permits unrestricted use, distribution, and reproduction in any medium, provided you give appropriate credit to the original author(s) and the source, provide a link to the Creative Commons license, and indicate if changes were made.

\section{References}

Akbari P, Braber S, Gremmels H et al (2014) Deoxynivalenol: a trigger for intestinal integrity breakdown. FASEB J 28:2414-2429

Amat C, Piqueras JA, Planas JM, Moret M (1999) Electrical properties of the intestinal mucosa of the chicken and the effects of luminal glucose. Poult Sci 78:1126-1131

Andretta I, Kipper M, Lehnen CR, Hauschild L, Vale MM, Lovatto PA (2011) Meta-analytical study of productive and nutritional interactions of mycotoxins in broilers. Poult Sci 90:1934-1940

Antonissen G, Van Immerseel F, Pasmans F et al (2014) The mycotoxin deoxynivalenol predisposes for the development of Clostridium perfringens-induced necrotic enteritis in broiler chickens. PLoS One 9:e108775

Antonissen G, Van Immerseel F, Pasmans F et al (2015) Mycotoxins deoxynivalenol and fumonisins alter the extrinsic component 
of intestinal barrier in broiler chickens. J Agri Food Chem 63(50):10846-10855

Aschenbach JR, Oswald R, Gäbel G (2000) Transport, catabolism and release of histamine in the ruminal epithelium of sheep. Pflugers Arch 440:171-178

Awad WA, Zentek J (2015) The feed contaminant deoxynivalenol affects the intestinal barrier permeability through inhibition of protein synthesis. Arch Toxicol 89:961-965

Awad WA, Böhm J, Razzazi-Fazeli E, Hulan HW, Zentek J (2004) Effects of deoxynivalenol on general performance and electrophysiological properties of intestinal mucosa of broiler chickens. Poult Sci 83:1964-1972

Awad WA, Böhm J, Razzazi-Fazeli E, Zentek J (2005a) In vitro effects of deoxynivalenol on electrical properties of the intestinal mucosa of laying hens. Poult Sci 84:921-927

Awad WA, Rehman H, Bohm J, Razzazi-Fazeli E, Zentek J (2005b) Effects of luminal deoxynivalenol and L-proline on electrophysiological parameters in the jejunums of laying hens. Poult Sci 84:928-932

Awad WA, Böhm J, Razzazi-Fazeli E, Zentek J (2006a) Effects of feeding deoxynivalenol contaminated wheat on growth performance, organ weights and histological parameters of the intestine of broiler chickens. J Anim Physiol Anim Nutr 90:32-37

Awad WA, Razzazi-Fazeli E, Böhm J, Ghareeb K, Zentek J (2006b) Effect of addition of a probiotic microorganism to broiler diets contaminated with deoxynivalenol on performance and histological alterations of intestinal villi of broiler chickens. Poult Sci 85:974-979

Awad WA, Aschenbach JR, Setyabudi FMCS, Razzazi-Fazeli E, Böhm J, Zentek J (2007a) In vitro effects of deoxynivalenol on small intestinal D-glucose uptake and absorption of deoxynivalenol across the isolated jejunal epithelium of laying hens. Poult Sci 86:15-20

Awad WA, Razzazi-Fazeli E, Böhm J, Zentek J (2007b) Influence of deoxynivalenol on the D-glucose transport across the isolated epithelium of different intestinal segments of laying hens. J Anim Physiol Anim Nutr 91:175-180

Awad WA, Ghareeb K, Böhm J, Zentek J (2010) Decontamination and detoxification strategies for the Fusarium mycotoxin deoxynivalenol in animal feed and the effectiveness of microbial biodegradation. Food Addit Contam 27:510-520

Awad WA, Hess M, Twarużek M et al (2011a) The impact of the Fusarium mycotoxin deoxynivalenol on the health and performance of broiler chickens. I J Mol Sci 12:7996-8012

Awad WA, Vahjen W, Aschenbach JR, Zentek J (2011b) A diet naturally contaminated with the Fusarium mycotoxin deoxynivalenol down regulates gene expression of glucose transporters in the intestine of broiler chickens. Livestock Sci 140:72-79

Awad WA, Ghareeb K, Böhm J (2012a) The toxicity of the Fusarium toxin deoxynivalenol in poultry feeding. World's Poult Sci J 68:651-668

Awad WA, Ghareeb K, Dadak A et al (2012b) Genotoxic effects of deoxynivalenol in broiler chickens fed with low protein diets. Poult Sci 91:550-555

Awad W, Ghareeb K, Böhm J, Zentek J (2013) The toxicological impacts of the Fusarium mycotoxin, deoxynivalenol, in poultry flocks with special reference to immunotoxicity. Toxins 5:912-925

Awad WA, Ghareeb K, Zentek J (2014a) Mechanisms underlying the inhibitory effect of the feed contaminant deoxynivalenol on glucose absorption in broiler chickens. Vet J 202:188-190

Awad WA, Ghareeb K, Dadak A, Hess M, Böhm J (2014b) Single and combined effects of deoxynivalenol mycotoxin and a microbial feed additive on lymphocyte DNA damage and oxidative stress in broiler chickens. PLoS One 9:e88028
Awad WA, Molnár A, Aschenbach JR et al (2015) Campylobacter infection in chickens modulates the intestinal epithelial barrier function. Innate Imm 21:151-160

Awad WA, Dublecz F, Hess C et al (2016a) Campylobacter jejuni colonization promotes the translocation of Escherichia coli to extraintestinal organs and disturbs the short-chain fatty acids profiles in the chicken gut. Poult Sci 95:2259-2265

Awad WA, Mann E, Dzieciol M et al (2016b) Age-related differences in the luminal and mucosa-associated gut microbiome of broiler chickens and shifts associated with Campylobacter jejuni infection. Front Cellul Infect Microbiol 6:154

Awad WA, Hess C, Hess M (2017) Enteric pathogens and their toxininduced disruption of the intestinal barrier through alteration of tight junctions in chickens. Toxins 9:60

Awad WA, Hess C, Hess M (2018) Re-thinking the chicken-Campylobacter jejuni interaction: a review. Avian Pathol 47:352-363

Dänicke S, Matthes S, Halle I, Ueberschär KH, Döll S, Valenta H (2003) Effects of graded levels of Fusarium toxin-contaminated wheat and of a detoxifying agent in broiler diets on performance, nutrient digestibility and blood chemical parameters. Brit Poult Sci 44:113-126

Ghareeb K, Awad WA, Soodoi C et al (2013) Effects of feed contaminant deoxynivalenol on plasma cytokines and mRNA expression of immune genes in the intestine of broiler chickens. PLoS One 8:e71492

Ghareeb K, Awad WA, Sid-Ahmed OE, Böhm J (2014) Insights on the host stress, fear and growth responses to the deoxynivalenol feed contaminant in broiler chickens. PLoS One 9:e87727

Ghareeb K, Awad WA, Böhm J, Zebeli Q (2015) Impacts of the feed contaminant deoxynivalenol on the intestine of monogastric animals: poultry and swine. J Appl Toxicol 35:327-337

Ghareeb K, Awad WA, Zebeli Q, Böhm J (2016) Deoxynivalenol in chicken feed alters the vaccinal immune response and clinical biochemical serum parameters but not the intestinal and carcass characteristics. J Anim Physiol Anim Nutr 100:53-60

Graziani F, Pinton P, Olleik H, Pujol A, Nicoletti C, Sicre M, Quinson N, Ajandouz EH, Perrier J, Pasquale ED, Oswald IP (2019) Maresca M (2019) Deoxynivalenol inhibits the expression of trefoil factors (TFF) by intestinal human and porcine goblet cells. Arch Toxicol. https://doi.org/10.1007/s00204-019-02425-6

Grenier B, Applegate TJ (2013) Invited review-modulation of intestinal functions upon mycotoxin ingestion: meta-analysis of published experiments in animals. Toxins 5:396-430

Groschwitz KR, Hogan SP (2009) Intestinal barrier function: molecular regulation and disease pathogenesis. J Allergy Clin Immunol 124:3-20

Kolf-Clauw M, Castellote J, Joly B et al (2009) Development of a pig jejunal explants culture for studying the gastrointestinal toxicity of themycotoxin deoxynialenol: histopathological analysis. Toxicol In Vitro 23:1580-1584

Kubena LF, Huff WE, Harvey RB, Corrier DE, Phillips TD, Creger CR (1988) Influence of ochratoxin A and deoxynivalenol on growing broiler chicks. Poult Sci 67:253-260

Kubena LF, Huff WE, Harvey RB, Phillips TD, Rottinghaus GE (1989) Individual and combined toxicity of deoxynivalenol and T-2 toxin in broiler chicks. Poult Sci 68:622-626

Lawhon SD, Maurer R, Suyemoto M, Altier C (2002) Intestinal shortchain fatty acids alter Salmonella typhimurium invasion gene expression and virulence through BarA/SirA. Mol Microbiol 46:1451-1464

Maresca M (2013) From the gut to the brain: journey and pathophysiological effects of the food-associated trichothecene mycotoxin deoxynivalenol. Toxins 5:784-820

Maresca M, Mahfoud R, Garmy N, Fantini J (2002) The mycotoxin deoxynivalenol affects nutrient absorption in human intestinal epithelial cells. J Nutr 132:2723-2731 
Maresca M, Yahi N, Younes-Sakr L, Boyron M, Caporiccio B, Fantini J (2008) Both direct and indirect effects account for the proinflammatory activity of enteropathogenic mycotoxins on the human intestinal epithelium: stimulation of interleukin-8 secretion, potentiation of interleukin-1beta effect and increase in the transepithelial passage of commensal bacteria. Toxicol Appl Pharmacol 228:84-92

Oakley BB, Lillehoj HS, Kogut MH et al (2014) The chicken gastrointestinal microbiome. FEMS Microbiol Lett 360:100-112

Odenwald MA, Turner JR (2013) Intestinal permeability defects: is it time to treat? Clin Gastroenterol Hepatol 11:1075-1083

Osselaere A, Santos R, Hautekiet V et al (2013) Deoxynivalenol impairs hepatic and intestinal gene expression of selected oxidative stress, tight junction and inflammation proteins in broiler chickens, but addition of an adsorbing agent shifts the effects to the distal parts of the small intestine. PLoS One 8:e69014

Pinton P, Oswald I (2014) Effect of deoxynivalenol and other type B trichothecenes on the intestine: a Review. Toxins 6:1615-1643

Pinton P, Nougayrede JP, Del Rio JC et al (2009) The food contaminant deoxynivalenol, decreases intestinal barrier permeability and reduces Claudin expression. Toxicol Appl Pharmacol 237:41-48
Pinton P, Graziani F, Pujol A, Nicoletti C, Paris O, Ernouf P, Di Pasquale E, Perrier J, Oswald IP, Maresca M (2015) Deoxynivalenol inhibits the expression by goblet cells of intestinal mucins through a PKR and MAP kinase dependent repression of the resistin-like molecule $\beta$. Mol Nutr Food Res 59:1076-1087

Tavelin S (2003) New approaches to studies of paracellular drug transport in intestinal epithelial cell monolayers. Acta Universitatis Upsaliensis. Comprehensive Summaries of Uppsala Dissertations from the Faculty of Pharmacy, Uppsala, 285, p 66, ISBN:91-554-5582-4

Vandenbroucke V, Croubels S, Martel A et al (2011) The mycotoxin deoxynivalenol potentiates intestinal inflammation by Salmonella typhimurium in porcine ileal loops. PLoS One 6:e23871

Publisher's Note Springer Nature remains neutral with regard to jurisdictional claims in published maps and institutional affiliations. 